\title{
Effects of Uric Acid on Lipid Levels in CKD Patients in a Randomized Controlled Trial
}

\author{
Rodney G. Bowden ${ }^{\mathrm{a}}$, Brian D. Shelmadine ${ }^{\mathrm{a}}$, Jennifer J. Moreillon ${ }^{\mathrm{a}}$, \\ Erika Deike $^{\mathrm{b}}$, Jackson O. Griggs ${ }^{\mathrm{c}}$, Ronald L. Wilson ${ }^{\mathrm{d}}$
}

\begin{abstract}
Background: Few studies have been conducted that compared lipid levels and uric acid in CKD or End-Stage Renal Disease (ESRD) patients with most using animal models. The purpose of the study was to explore effects on lipids while controlling uric acid levels in CKD patients.

Methods: Twenty-four CKD patients $(\mathrm{N}=24)$ volunteered to participate in this study. The study was conducted using a doubleblind, randomized, placebo controlled experimental protocol. The experimental group was prescribed $300 \mathrm{mg}$ of allopurinol PO daily by their treating physician and followed prospectively for 8-weeks. The control group consumed a similar pill once a day for 8-weeks.

Results: ANCOVA revealed significant differences in total cholesterol $(\mathrm{P}=0.009)$ and Apo $\mathrm{B}(\mathrm{P}=0.006)$ with lower levels in the allopurinol group. A trend emerged with $\operatorname{LDL}(\mathrm{P}=0.052)$ with lower levels in the allopurinol group. No significant differences were discovered in triglycerides $(\mathrm{P}=0.403)$, HDL $(\mathrm{P}=0.762)$ and total Cholesterol/HDL Ratio $(\mathrm{P}=0.455)$.

Conclusions: After statistically controlling for compliance and inflammation significant differences between groups were observed for total cholesterol and Apo B. In both instances the allopurinol group had lower concentrations than the placebo group. Similarly, a trend was observed in LDL with the allopurinol group having lower concentrations than the placebo group.
\end{abstract}

\footnotetext{
Manuscript accepted for publication April 5, 2013

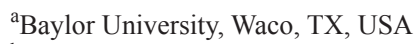

${ }^{\mathrm{b}}$ Texas Lutheran University, Austin, Texas, USA

${ }^{c}$ Family Medicine, Family Health Center. Waco, Texas, USA

${ }^{\mathrm{d} C e n t r a l}$ Texas Nephrology Associates, Waco, Texas, USA

${ }^{\text {e}}$ Corresponding author: Rodney G. Bowden, Baylor University, Associate Dean for Graduate Studies and Research, Professor of Health Education, Baylor University, Waco, TX.

Email: Rodney_Bowden@baylor.edu

doi: http://dx.doi.org/10.4021/cr263w
}

Keywords: Uric acid; Cholesterol; Chronic kidney disease; LDL; HDL

\section{Introduction}

Allopurinol is a xanthine oxidase inhibitor that effectively blocks the formation of uric acid and is used to treat gout. However, high concentrations of uric acid (hyperuricemia; $\geq 7 \mathrm{mg} / \mathrm{dL}$ in men and $\geq 6 \mathrm{mg} / \mathrm{dL}$ in women) have previously been linked to components of metabolic syndrome [14], have been proposed to have a role in developing diabetic nephropathies [5], and have been implicated in the development of hypertension and a decrease in renal function [6].

Lowering uric acid levels using allopurinol has been demonstrated to improve glomerular filtration rate (GFR), blood pressure (BP) and c-reactive protein (CRP) levels in patients with normal renal function [6]. In patients with chronic kidney disease (CKD), lowering uric acid has been reported to significantly improve CRP levels, independently slow the progression of renal disease, and reduce cardiovascular events by 71\% [7]. Few studies have been conducted that compared lipid levels and uric acid in CKD or End-Stage Renal Disease (ESRD) patients with most using animal models. Nakagawa et al [8] and Khosla et al [9] reported that decreased uric acid through the use of allopurinol decreased serum lipid levels and triglycerides in rats. Additionally, allopurinol used in a pilot study of ESRD patients significantly improved LDL cholesterol and triglyceride levels [10] although earlier research in past decades did not find allopurinol to be effective at reducing serum levels of cholesterol [11, 12]. Yet, other study authors [13] have reported that uric acid plays a role in dyslipidemia through the suppression of lipid peroxidation.

Complete mechanisms for the effectiveness of allopurinol in improving CRP, BP, GFR, lipid and triglyceride levels remain elusive. However, some proposed mechanisms include suppression of lipid peroxidase [13] and decreases in critical lipase activity [8]. Additionally, allopurinol is known to scavenge free radicals and reduce inflammation [13]. Therefore, the purpose of this study was to explore effects 
Table 1. Demographic and Pretest Values for Treatment and Control Groups

\begin{tabular}{|c|c|c|c|}
\hline Variable & $\begin{array}{l}\text { Allopurinol Group (n } \\
=12 \text { ) }\end{array}$ & $\begin{array}{l}\text { Placebo Group (n } \\
=12)\end{array}$ & P-value \\
\hline Gender, n (\%) & & & 0.667 \\
\hline Females & $3(25.0)$ & $7(58.3)$ & \\
\hline Males & $9(75.0)$ & $5(41.7)$ & \\
\hline Age, y (SD) & $59.2(10.1)$ & $62.4(9.5)$ & 0.307 \\
\hline Ethnicity, n (\%) & & & 0.197 \\
\hline African American & $4(33.3)$ & $8(66.7)$ & \\
\hline Caucasian & $4(33.3)$ & $1(8.3)$ & \\
\hline Hispanic & $4(33.3)$ & $3(25.0)$ & \\
\hline Weight (kg), mean (SD) & $86.31(14.10)$ & $88.35(31.54)$ & 0.138 \\
\hline Systolic BP, mean (SD) & $142.17(14.30)$ & $139.25(30.07)$ & 0.764 \\
\hline Diastolic BP, mean (SD) & $76.17(9.97)$ & $73.67(15.92)$ & 0.649 \\
\hline GFR, mean (SD) & $47.30(15.90)$ & $45.10(19.80)$ & 0.503 \\
\hline Diabetes, n (\%) & $8(66.7)$ & $5(41.7)$ & 0.154 \\
\hline CRP, mean (SD) & $7.73(2.06)$ & $8.87(3.18)$ & 0.717 \\
\hline IL-6, mean (SD) & $21.76(14.43)$ & $17.38(12.46)$ & 0.327 \\
\hline TNF- $\alpha$, mean (SD) & $22.10(9.91)$ & $23.33(11.76)$ & 0.703 \\
\hline Albumin, mean (SD) & $4.24(0.24)$ & $4.11(0.43)$ & 0.445 \\
\hline
\end{tabular}

CRP, uric acid, and albumin are measured in mg/dL; IL-6, IL-1 $\beta$, and TNF- $\alpha$ are measured in $\mathrm{pg} / \mathrm{mL}$.

on lipids while controlling uric acid levels by means of allopurinol use in CKD patients.

\section{Methods}

\section{Patients}

Twenty-four CKD patients $(\mathrm{N}=24)$ from a family medicine clinic volunteered to participate in this study after being contacted by their primary care physician. Participants completed a medical history questionnaire and completed a general examination by their treating physician to determine whether they met eligibility criteria. Patients were excluded from the study if they were under 18 years of age, had an active illness requiring hospitalization, had a previous allergic reaction to allopurinol or any of its components, positive blood tests for Hepatitis B and C, dementia, malabsorption syndromes, were pregnant, had a life expectancy of less than three months, malignant hypertension, and/or a history of medication non-compliance. Approval to conduct the study was provided by university and clinic institutional review boards. Patients meeting eligibility requirements signed informed consent statements.

\section{Experimental design}

The study was conducted using a double-blind, randomized, placebo controlled experimental design with 12 patients randomly placed in the experimental group and 12 patients randomly placed in the control group for a sample size of twenty-four. All participants completed the study. The experimental group was prescribed $300 \mathrm{mg}$ of allopurinol PO daily by their treating physician and followed prospectively for 8 weeks. The control group consumed a similar pill once a day for 8 weeks that contained rice flour. Patients were monitored weekly by researchers conducting the study through phone consultation and through regularly scheduled 
Table 2. Pretest (t-Test) and Posttest (ANCOVA) Lipid Values for the Allopurinol Group and the Control Group

\begin{tabular}{|c|c|c|c|}
\hline Variable* & $\begin{array}{l}\text { Allopurinol Group (n } \\
=12)\end{array}$ & $\begin{array}{l}\text { Placebo Group (n } \\
=12)\end{array}$ & P-value \\
\hline Pre HDL & $39.08(13.48)$ & $35.75(7.30)$ & 0.452 \\
\hline Post HDL & $36.40(14.72)$ & $38.30(9.63)$ & 0.762 \\
\hline Pre LDL & $97.00(40.68)$ & $114.92(34.77)$ & 0.707 \\
\hline Post LDL & $96.10(32.46)$ & $119.56(42.05)$ & 0.052 \\
\hline Pre Triglycerides & $126.83(42.88)$ & $135.25(75.27)$ & 0.408 \\
\hline Post Triglycerides & $161.20(89.67)$ & $176.50(117.96)$ & 0.403 \\
\hline Pre Total Cholesterol & $161.42(38.23)$ & $177.58(41.13)$ & 0.741 \\
\hline Post Total cholesterol & $164.80(35.97)$ & $188.00(44.46)$ & 0.009 \\
\hline Pre Total Cholesterol- HDL Ratio & $4.55(1.92)$ & $5.21(1.88)$ & 0.405 \\
\hline Post Total Cholesterol- HDL Ratio & $5.06(2.15)$ & $5.18(1.70)$ & 0.455 \\
\hline Pre Apolipoprotein B & $78.58(25.96)$ & $92.25(22.86)$ & 0.886 \\
\hline Post Apolipoprotein B & $79.30(24.15)$ & $100.30(25.46)$ & 0.006 \\
\hline Pre Uric Acid & $7.73(2.06)$ & $8.87(3.18)$ & 0.307 \\
\hline Post Uric Acid & $6.50(1.78)$ & $8.61(3.30)$ & 0.034 \\
\hline Pre GFR & $47.30(15.90)$ & $45.10(19.80)$ & 0.407 \\
\hline Post GFR & $45.19(13.56)$ & $41.15(18.76)$ & 0.578 \\
\hline Pre Creatinine & $1.57(0.61)$ & $2.10(0.88)$ & 0.307 \\
\hline Post Creatinine & $1.62(.64)$ & $2.11(0.97)$ & 0.185 \\
\hline
\end{tabular}

CRP, uric acid, and albumin are measured in $\mathrm{mg} / \mathrm{dL} ; \mathrm{IL}-6, \mathrm{IL}-1 \beta$, and TNF- $\alpha$ are measured in $\mathrm{pg} / \mathrm{mL}$.

appointments to see their treating physician to measure compliance in both the experimental and control groups for the entire length of the study. Compliance in both groups was monitored through the standard practice of pill counting with patients returning their unused medication/placebo pills at the end of the study with those consuming $80 \%$ considered compliant $[14,15]$. Additionally, compliance with allopurinol was measured through uric acid levels and participants in the experimental group were considered compliant if their uric acid levels decreased by at least $20 \%$ during the study [8]. Ten $(83.3 \%)$ of participants in the experimental group were considered compliant based on a decrease in uric acid of $20 \%$ or greater, while seven $(58.3 \%)$ were compliant in the control group based on consuming greater than $80 \%$ of their pills (placebo). Overall seventeen $(70.8 \%)$ of participants were compliant. A sample size of greater than 23 was chosen to ensure a power of greater than $80 \%$ based on formulas published by Freedman [16] in which the alpha level is .05 and the average rate of change in uric acid values is $20 \%$.

\section{Data collection}

Following a 12-hour fast approximately 50 milliliters of blood was collected for analysis at baseline and 8 weeks. Samples were analyzed for levels of uric acid, albumin, inflammatory markers of CRP, Tumor Necrosis Factor-alpha (TNF- $\alpha$ ), interleukin-6 (IL-6), triglycerides, total cholester- 


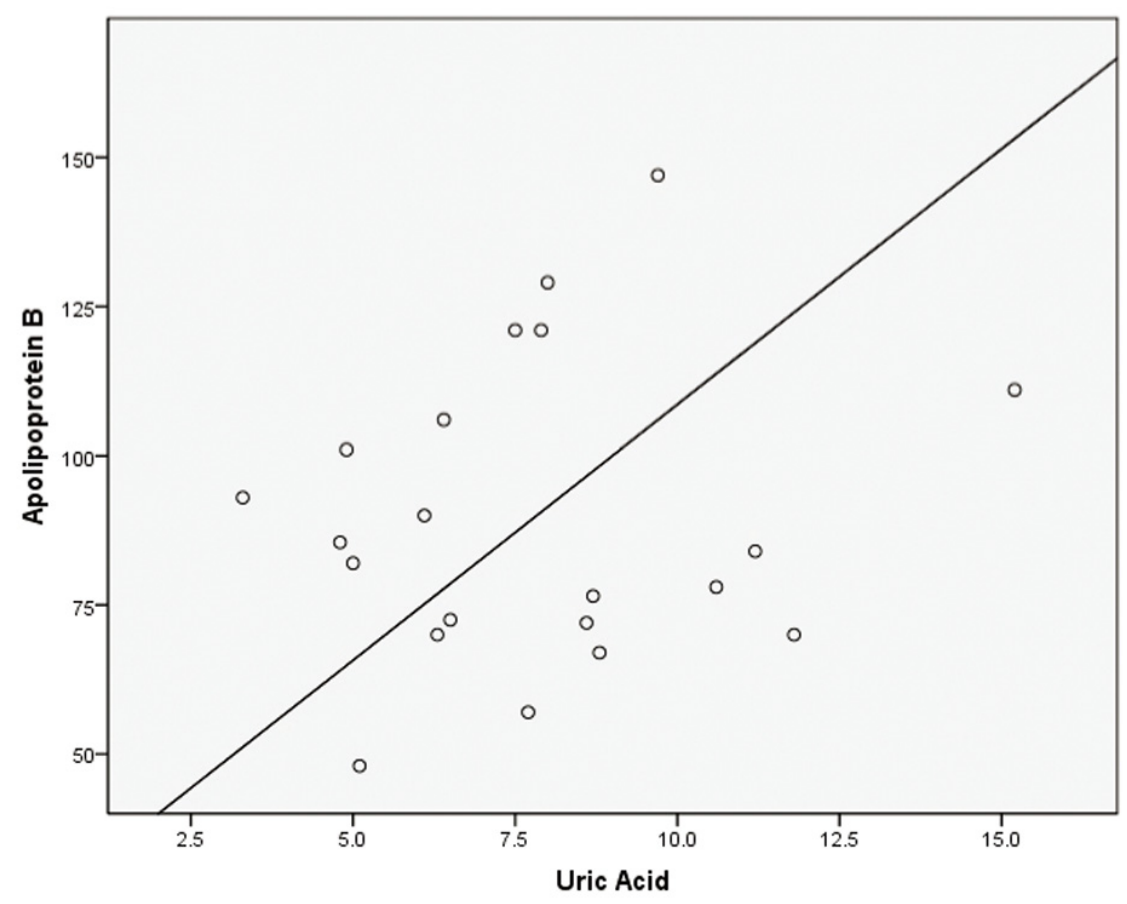

Figure 1. Simple scatterplot for uric acid and apolipoprotein B.

ol, high-density lipoprotein cholesterol (HDL), low-density lipoprotein cholesterol (LDL), total cholesterol/HDL ratio, and apolipoprotein B (ApoB). Blood samples for inflammatory markers was centrifuged within 10 minutes at 2,400 rpm for 10 minutes and stored at $-20^{\circ} \mathrm{C}$. Plasma levels were analyzed for TNF- $\alpha$, IL- 6 , and CRP using commercially available assays with an iMark microplate reader (Bio-Rad Laboratories, Inc., Hercules, CA). All assays were performed in duplicate using the manufacturer recommended wavelength against a known standard curve depending on the specifications of the protocol. Standard protocol was used with commercially available kits from Cayman Chemical (Ann Arbor, MI) for each cytokine. Lipid profiles (triglycerides, total cholesterol, HDL, LDL, ApoB, and total cholesterol/ HDL), albumin levels, and CRP were independently analyzed by Quest Diagnostics (Dallas, TX). Possible adverse events with allopurinol were tracked through the treating physician with no side effects reported by study participants. Additionally there were no changes in ALT, AST, alkaline phosphatase, albumin, and bilirubin during the study.

\section{Statistical analysis}

Independent t-tests were used to ascertain differences at baseline between groups for uric acid, age, gender, race, albumin, CRP, IL-6, TNF- $\alpha$, LDL, HDL, triglycerides, total cholesterol, total cholesterol/HDL ratio and ApoB. ANCOVA was used to measures differences at 8 weeks between groups for all lipid variables after controlling for known covariates of albumin, CRP, IL- 6 , TNF- $\alpha$, and medication/ placebo compliance. ANCOVA was used to control for variables that could have affected the outcome of lipids but were not a variable of interest. An independent sample Kolmogorov-Smirnov test of normality was conducted on uric acid. Pearson correlations were calculated between uric acid and lipid variables of total cholesterol, ApoB and LDL. Statistical analyses were performed using SPSS for Windows version 18.0 (SPSS Inc., Chicago, IL). All data is presented as mean \pm standard deviation (SD) with $\mathrm{P}<0.05$ considered significant.

\section{Results}

Independent t-tests revealed no significant differences at baseline for uric acid $(\mathrm{P}=0.307)$, age $(\mathrm{P}=0.435)$, albumin $(\mathrm{P}=0.445), \mathrm{CRP}(\mathrm{P}=0.717), \mathrm{IL}-6(\mathrm{P}=0.327), \mathrm{TNF}-\alpha(\mathrm{P}=$ $0.703)$, triglycerides $(\mathrm{P}=0.408)$, total cholesterol $(\mathrm{P}=0.540)$, HDL $(\mathrm{P}=0452)$, LDL $(\mathrm{P}=0.707)$, total cholesterol/HDL ratio $(P=0.741)$, and ApoB $(P=0.886)$. Chi Square revealed no significant differences in gender $(\mathrm{P}=0.667)$ and race $(\mathrm{P}$ $=0.197$ ) between groups. An independent sample Kolmogorov-Smirnov test of normality for uric acid revealed a normal distribution $(\mathrm{P}=0.518)$. Results are presented in Table 1 .

ANCOVA revealed significant differences in total cholesterol $(\mathrm{P}=0.009)$ and Apo B $(\mathrm{P}=0.006)$ with lower levels in the allopurinol group. A trend emerged with LDL $(\mathrm{P}=$ 0.052 ) with lower levels in the allopurinol group. No signifi- 


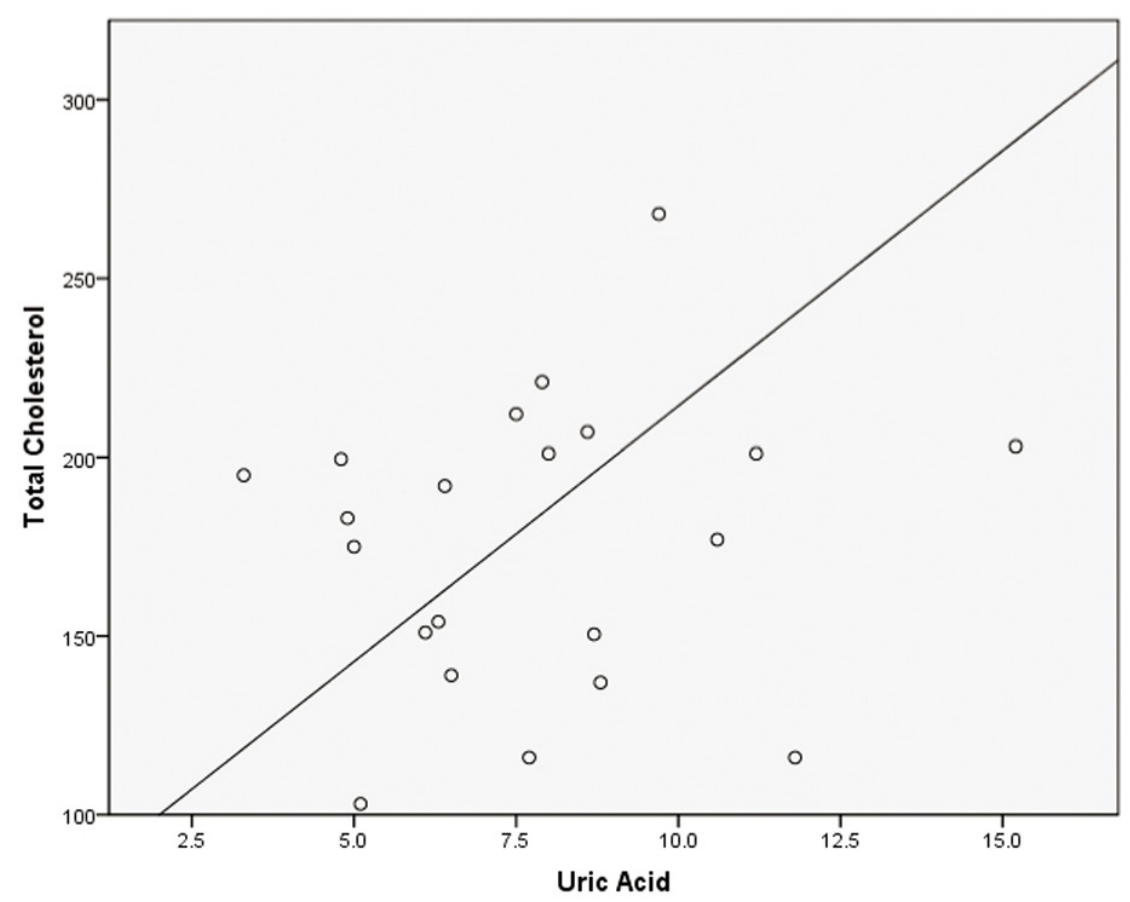

Figure 2. Simple scatterplot for total cholesterol and uric acid.

cant differences were discovered in triglycerides $(\mathrm{P}=0.403)$, HDL $(\mathrm{P}=0.762)$, total Cholesterol/HDL Ratio $(\mathrm{P}=0.455)$ and uric acid $(\mathrm{P}=0.185)$ between groups. Results are presented in Table 2.

Pearson correlations were calculated with posttest values revealing week correlations between uric acid and total cholesterol $\left(r^{2}=0.100\right)$, uric acid and ApoB $\left(r^{2}=0.142\right)$, and uric acid and LDL $\left(\mathrm{r}^{2}=0.121\right)$ Scatterplots were created using posttest uric acid and posttest lipid values that were discovered to be statistically different with results presented in Figure 1-3.

\section{Discussion}

Hyperuricemia has been linked to an increased risk for cardiovascular disease in patients with normal renal function and an accelerated progression of chronic kidney disease [6, 10]. While there is still discussion as to whether uric acid is a marker of increased risk or whether it serves a causative role [17], recent evidence indicates uric acid may in fact have a causative role [1]. However, most research has focused on mechanisms that may lead to hypertension and/or increased levels of inflammation through lowering uric acid levels [6]. Only a handful have sought to explore the effects of lowering uric acid on lipid levels as studies show elevated lipid levels in hyperuricemic patients with both normal [6] and impaired renal function [10] and obese patients [18] further providing a link to hyperuricemia and metabolic syndrome.
Therefore, the purpose of this study was to explore effects on lipids while controlling uric acid levels by means of allopurinol use in CKD patients. After controlling for compliance, albumin, CRP, IL-6, and TNF- $\alpha$ significant differences between groups after eight weeks were observed for total cholesterol and Apo B. In both instances the allopurinol group had lower concentrations than the placebo group. Similarly, a trend was observed in LDL with the allopurinol group having lower concentrations than the placebo group. However, no significant differences were observed between the groups at baseline. These findings support previous research in humans [10] and animal models [8, 9].

The most interesting finding is the reduction $\mathrm{ApoB}$ in the experimental group. The Third Report of the National Cholesterol Education Program Expert Panel on Detection, Evaluation, and Treatment of High Blood Cholesterol in Adults [19] suggests that the reduction of LDL is important but must also be accompanied by reductions in LDL particle numbers and smaller LDL particles. ApoB is an indirect measure of LDL particle number and suggests the reduction of uric acid may play a role in helping to produce fewer LDL particle numbers and fewer small and dense atherogenic LDL particles. It should be noted that a trend emerged with LDL that also demonstrated a decrease in LDL cholesterol with a decrease in uric acid. Additionally, triglycerides increased in both groups which is supported by previous research [10] and may be associated with an increase in lipoprotein lipase (LPL) to help control LDL particle number and LDL but may have not been enough to overcome advanced CKD disease and the effects of gout with both disease states normally ex- 


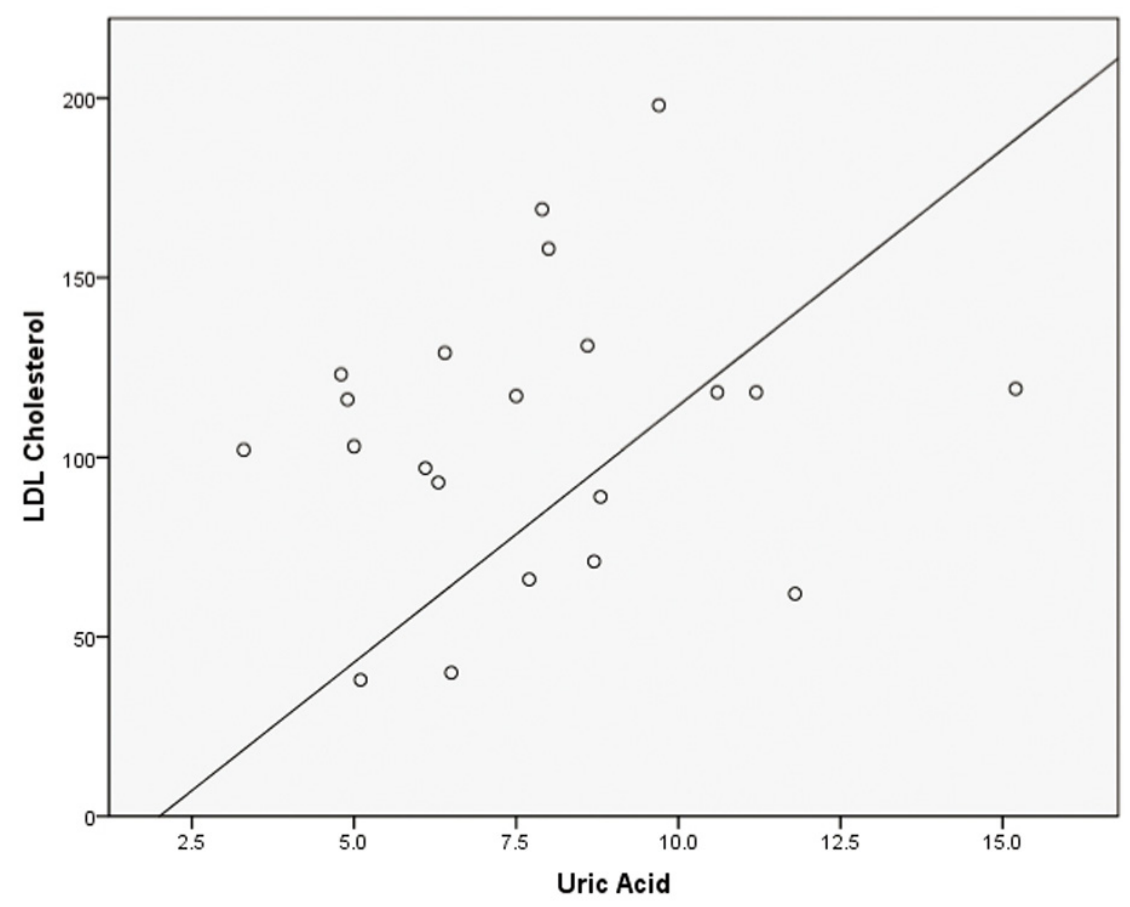

Figure 3. Simple scatterplot for LDL and uric acid.

periencing elevated triglycerides [10]. LPL is the principle enzyme associated with lipolysis of triglycerides and may be affected by high levels of inflammation in this study population [20]. Though small and dense LDL particles are normally associated with elevated triglycerides, the findings in this study and other study authors suggest the decrease of uric acid may play a role in the enzymatic activity of LPL $[10,12,17]$. Uric acid is major cause of oxidative stress, reduced nitrous oxide release and combined with an increase in activity of LPL may cause higher lipid levels and particle numbers [20]. It should be noted that gout is associated with oxidative stress and LDL plays a significant role in the progression of heart disease in both healthy and gout populations [21]. Krishnan et al [21] reports that uric acid can act as pro-oxidant or an antioxidant, which is dependent upon the state of the oxidized lipoprotein. The authors further suggest that when LDL is mildly oxidized uric acid becomes a pro-oxidant and may be related to lipid hyperoxides. Iso-O et al [22] suggests that oxidative stress can induce the peroxidation of both lipids and lipid hyperoxides which can then produce oxidative stress in the cell. Uric acid when combined with oxidized LDL may cause there to be increases in ApoB and LDL particle numbers and the reason why the use of allopurinol can help decrease some lipoproteins. Yet the finding of both an increase in triglycerides and a decrease in $\mathrm{ApoB}$ does not correspond well with previous studies in non-CKD patients [23].

Another important finding of this study was the decrease in total cholesterol in allopurinol group. Though LDL is a strong predictor of heart disease, total cholesterol is also an independent predictor of risk. Previous findings have suggested that hyperuricemia occurs in patients with ESRD and the comorbid condition of gout. High uric acid levels have been associated with insulin resistance, hypertension, endothelial dysfunction, and renal impairment as well as in dyslipidemia, especially in patients with renal impairment [6]. Furthermore hyperuricemia is thought to impair endothelium dependent vasodilatation primarily through lipid oxidation that can cause an increase in total cholesterol [20]. Finally, Ellestad et al [17] suggest that hyperuricemia is a reflection of the degree of xanthine oxidase activation which is derived from oxygen free radical production. This cascade of events might be associated with an increase in total cholesterol suggesting allopurinol as means to control total cholesterol. Our study supports these findings indirectly as total cholesterol values were lower in the group that had lower uric acid levels through means of allopurinol use.

Though the findings of no significant changes in inflammatory markers was not the primary outcome of our study they are not in congruence with previous literature indicating that treatment of hyperuricemia with allopurinol reduces inflammation [24]. Differences in results may be due to the short duration of study as well as the population studied. Additionally, high inflammation levels are associated with a decrease in HDL which may help explain our non-significant findings with HDL [20]. The duration of the current study, eight weeks, may not have provided adequate time to affect inflammation levels. Additionally, CKD patients often have 
a myriad of co-morbidities and complications, such as diabetes or coronary heart disease that may have hindered the effect of lowering uric acid levels on inflammation.

A few limitations existed for this study and are worthy of note. The sample size was small making inferences more challenging in finding significance and evident trends. Also, the small sample size can lead to Type I errors with an over or underestimation of results. Secondly, some patients presented with Type II diabetes which may have been a confounding variable. By controlling for known confounding variables a clearer picture was presented regarding cholesterol and uric acid. Future studies should include a larger sample size with patients followed for a longer study period. Future studies will help to demonstrate if uric acid is simply a confounding variable or plays a causative role in controlling lipid levels.

\section{Conclusions}

The significant differences in total cholesterol and Apo B as well as the trend in LDL concentrations are novel findings suggesting that compliant patients ingesting $300 \mathrm{mg} /$ day of allopurinol may experience a decrease in lipid levels and reduction in heart disease risk. This novel approach for controlling lipids in CKD may provide another means to reduce heart disease risk in a population that has a rate of dyslipidemia that has been reported to be triple of apparently healthy populations [21].

\section{Acknowledgement}

We would like to thank the entire staff at the Family Health Center for making this study possible, especially CraShanta Evans, Toni Marshall, and Linda Garcia.

\section{Conflict of Interest Statement}

No authors have a conflict of interest.

\section{References}

1. Chen LY, Zhu WH, Chen ZW, Dai HL, Ren JJ, Chen JH, Chen LQ, et al. Relationship between hyperuricemia and metabolic syndrome. J Zhejiang Univ Sci B. 2007;8(8):593-598.

2. Choi HK, Ford ES. Prevalence of the metabolic syndrome in individuals with hyperuricemia. Am J Med. 2007;120(5):442-447.

3. Emmerson B. Hyperlipidaemia in hyperuricaemia and gout. Ann Rheum Dis. 1998;57(9):509-510.

4. Lin JD, Chiou WK, Chang HY, Liu FH, Weng HF. Serum uric acid and leptin levels in metabolic syndrome: a quandary over the role of uric acid. Metabolism. 2007;56(6):751-756.

5. Hovind P, Rossing P, Johnson RJ, Parving HH. Serum uric acid as a new player in the development of diabetic nephropathy. J Ren Nutr. 2011;21(1):124-127.

6. Kanbay M, Ozkara A, Selcoki Y, Isik B, Turgut F, Bavbek $\mathrm{N}, \mathrm{Uz}$ E, et al. Effect of treatment of hyperuricemia with allopurinol on blood pressure, creatinine clearence, and proteinuria in patients with normal renal functions. Int Urol Nephrol. 2007;39(4):1227-1233.

7. Goicoechea M, de Vinuesa SG, Verdalles U, Ruiz-Caro C, Ampuero J, Rincon A, Arroyo D, et al. Effect of allopurinol in chronic kidney disease progression and cardiovascular risk. Clin J Am Soc Nephrol. 2010;5(8):13881393.

8. Nakagawa T, Hu H, Zharikov S, Tuttle KR, Short RA, Glushakova O, Ouyang X, et al. A causal role for uric acid in fructose-induced metabolic syndrome. Am J Physiol Renal Physiol. 2006;290(3):F625-631.

9. Khosla UM, Zharikov S, Finch JL, Nakagawa T, Roncal C, Mu W, Krotova K, et al. Hyperuricemia induces endothelial dysfunction. Kidney Int. 2005;67(5):17391742.

10. Shelmadine B, Bowden RG, Wilson RL, Beavers D, Hartman J. The effects of lowering uric acid levels using allopurinol on markers of metabolic syndrome in end-stage renal disease patients: a pilot study. Anadolu Kardiyol Derg. 2009;9(5):385-389.

11. Gibson T, Grahame R. Gout and hyperlipidaemia. Ann Rheum Dis. 1974;33(4):298-303.

12. Ulreich A, Kostner GM, Pfeiffer KP, Sedlmayr P, Rainer F. Serum lipids and lipoproteins in patients with primary gout. Rheumatol Int. 1985;5(2):73-77.

13. Minami M, Ishiyama A, Takagi M, Omata M, Atarashi K. Effects of allopurinol, a xanthine oxidase inhibitor, on renal injury in hypercholesterolemia-induced hypertensive rats. Blood Press. 2005;14(2):120-125.

14. Jasti S, Siega-Riz AM, Cogswell ME, Hartzema AG, Bentley ME. Pill count adherence to prenatal multivitamin/mineral supplement use among low-income women. J Nutr. 2005;135(5):1093-1101.

15. Lee JY, Kusek JW, Greene PG, Bernhard S, Norris $\mathrm{K}$, Smith D, Wilkening B, et al. Assessing medication adherence by pill count and electronic monitoring in the African American Study of Kidney Disease and Hypertension (AASK) Pilot Study. Am J Hypertens. 1996;9(8):719-725.

16. Freedman LS. Tables of the number of patients required in clinical trials using the logrank test. Stat Med. 1982;1(2):121-129.

17. Ellestad MH. Xanthine oxidase inhibitors the unappreciated treatment for heart failure. Cardiovasc Hematol Disord Drug Targets. 2007;7(4):291-294.

18. Gagliardi AC, Miname MH, Santos RD. Uric acid: A 
marker of increased cardiovascular risk. Atherosclerosis. 2009;202(1):11-17.

19. Third Report of the National Cholesterol Education Program (NCEP) Expert Panel on Detection, Evaluation, and Treatment of High Blood Cholesterol in Adults (Adult Treatment Panel III) final report. Circulation. 2002;106(25):3143-3421.

20. Grigore, C, Stoian, I, Grigore, O, Dawkins, L, Isacoff1, D, Bruckner, I: Oxidative Stress, Uric Acid, Vascular Inflammation in Non-Smoking Metabolic Syndrome Patients. Int J Biol Biom Eng. 2010;3:61-9. .

21. Krishnan E. Inflammation, oxidative stress and lipids: the risk triad for atherosclerosis in gout. Rheumatology
(Oxford). 2010;49(7):1229-1238.

22. Iso ON, Noto H, Hara M, Togo M, Karasawa K, Ohashi $\mathrm{N}$, Noiri E, et al. Adenovirus-mediated gene transfer and lipoprotein-mediated protein delivery of plasma PAF$\mathrm{AH}$ ameliorates proteinuria in rat model of glomerulosclerosis. Mol Ther. 2006;13(1):118-126.

23. Suliman ME, Johnson RJ, Garcia-Lopez E, Qureshi AR, Molinaei H, Carrero JJ, Heimburger O, et al. J-shaped mortality relationship for uric acid in CKD. Am J Kidney Dis. 2006;48(5):761-771.

24. Becker MA, Jolly M. Hyperuricemia and associated diseases. Rheum Dis Clin North Am. 2006;32(2):275-293, V-vi. 